\title{
Carrier Tunneling in High-Frequency Electric Fields
}

\author{
S. D. Ganichev, ${ }^{1,2}$ E. Ziemann, ${ }^{1}$ Th. Gleim, ${ }^{1}$ W. Prettl, ${ }^{1}$ I. N. Yassievich, ${ }^{2}$ V. I. Perel, ${ }^{2}$ I. Wilke,,${ }^{3, *}$ and E. E. Haller ${ }^{3}$ \\ ${ }^{1}$ Institut für Experimentelle und Angewandte Physik, Universität Regensburg, 93040 Regensburg, Germany \\ ${ }^{2}$ A.F. Ioffe Physicotechnical Institute, Russian Academy of the Sciences, St. Petersburg, 194021, Russia \\ ${ }^{3}$ Materials Sciences Division, Lawrence Berkeley National Laboratory and University of California, Berkeley, California 94720
}

(Received 31 July 1997)

\begin{abstract}
An enhancement of tunnel ionization of deep impurities in semiconductors in an alternating field as compared to static fields has been observed. The transition between the quasistatic and the highfrequency regime is determined by the tunneling time. For the case of deep impurities this is the time of redistribution of the defect vibrational system which depends strongly on temperature and the impurity structure. A theory of tunnel ionization of deep impurities by high-frequency fields has been developed. [S0031-9007(98)05504-5]
\end{abstract}

PACS numbers: 71.55.-i, 72.20.Ht, 72.40.+w

The effect of high-frequency coherent radiation on tunneling in semiconductor superlattices and nanostructures has attracted considerable attention recently. The superposition of a static electric field and an alternating field causes a wealth of new phenomena as a result of photon assisted tunneling [1-3]. In all these cases tunneling is accomplished by a static electric field, and the radiation influences the barrier penetration probability. An intense radiation field, however, can, in fact, both generate the tunneling barrier and initiate tunneling. Such a tunneling process has been observed with the result that the highfrequency field acts like a static field and the tunneling probability does not depend on frequency [4]. The frequency independent tunneling, however, must be limited to frequencies $\Omega$ with $\Omega \tau<1$, where $\tau$ is the tunneling time. This has been shown in a number of theoretical works [5-10], but has never been explored experimentally. In contrast to static electric fields where the electron tunnels at a fixed energy, in alternating fields the energy of the electron is not conserved during tunneling. In this case the electron can absorb energy from the field, which should lead to a sharp increase of the tunneling probability with increasing frequency for $\Omega \tau>1$.

Here we report on the first experimental demonstration of this effect observed in tunneling ionization of deep impurities in semiconductors. We show that the transition from the quasistatic regime $\Omega \tau<1$ to the high-frequency regime $\Omega \tau>1$ occurs at terahertz frequencies. In the quasistatic regime the electron tunnels at the momentary magnitude of the electric field in a time shorter than the period of oscillation, thus the electric field acts like a static field. The ionization probability is independent of frequency and increases with rising field strength $E$ like $\exp \left(E^{2} / E_{c}^{2}\right)$ where $E_{c}$ is a characteristic field [4]. In the high-frequency regime the ionization probability, being characterized by the same field dependence, substantially increases with increasing frequency. In contrast to tunneling ionization of atoms, where only electron tunneling takes place [5], ionization of impurities in solids is accomplished by two simultaneous tunneling processes, electron tunneling and the redistribution of the vibrational system by defect tunneling. A theory of the process is developed showing for the first time that in this case the electron tunneling time is controlled by defect tunneling and both tunneling times are equal.

The measurements have been carried out on different types of deep impurities: substitutional impurities with weak electron-phonon coupling $(\mathrm{Ge}: \mathrm{Hg}, \mathrm{Ge}: \mathrm{Cu}$ with acceptor density in the range $10^{14}-10^{15} \mathrm{~cm}^{-3}$ ) and autolocalized $\mathrm{DX}^{-}$centers, with strong electron-phonon coupling $\left(\mathrm{Al}_{x} \mathrm{Ga}_{1-x} \mathrm{As}\right.$ :Te, $\mathrm{Al}_{x} \mathrm{Ga}_{1-x} \mathrm{Sb}$ :Te with $x=0.35$ and 0.5 ). In the investigated temperature range from 4.2 to $80 \mathrm{~K}$, for autolocalized impurities up to $150 \mathrm{~K}$, practically all impurities are occupied in thermal equilibrium. Tunnel ionization has been achieved by far-infrared laser radiation with photon energies much smaller than the thermal impurity ionization energy $\varepsilon_{T}$. The ionization probability has been determined by detecting photoconductivity.

The radiation sources used were a pulsed far-infrared molecular laser optically pumped by a TEA-CO $\mathrm{CO}_{2}$ laser and the Santa Barbara free-electron laser (FEL). Using $\mathrm{NH}_{3}$ as active gas for the optically pumped laser, $40 \mathrm{~ns}$ pulses with intensity up to $2 \mathrm{MW} / \mathrm{cm}^{2}$ have been obtained at frequencies $\Omega$ of $25,21,13$, and $6.7 \mathrm{THz}$, respectively. The operating parameters of the FEL are pulse duration $6 \mu \mathrm{s}$, intensity up to $1 \mathrm{~kW} / \mathrm{cm}^{2}$, and frequencies $6.8,4.5$, and $3.4 \mathrm{THz}$.

Ionization of deep impurities by far-infrared radiation has been observed for all samples in the whole frequency range investigated. In a wide range of temperature, frequency and electric field strength ionization can be attributed to phonon assisted tunneling [4]. Experimental results obtained with AlGaAs:Te at $T=150 \mathrm{~K}$ are shown in Fig. 1(a). The ratio of the conductivity under irradiation to the dark conductivity $\sigma_{i} / \sigma_{d}$, which is proportional to the ionization probability, is plotted as a function of the square of the peak electric field strength $E$. The ionization probability at this temperature and this material is independent of radiation frequency and increases with rising $E$ like $\exp \left(E^{2} / E_{c}^{2}\right)$. Such a behavior has been 


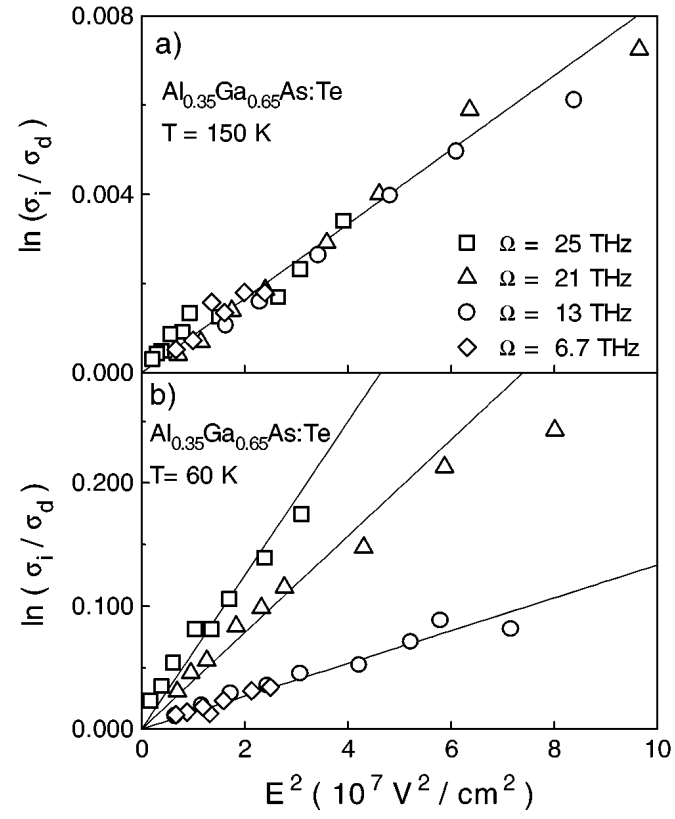

FIG. 1. Dependence of $\ln \left(\sigma_{i} / \sigma_{d}\right)$ for $\mathrm{DX}^{-}$centers in $\mathrm{Al}_{0.35} \mathrm{Ga}_{0.65} \mathrm{As}$ on the square of the amplitude of the electric field of the radiation at different frequencies and for $T=150 \mathrm{~K}$ (a) and $T=60 \mathrm{~K}$ (b). The photoconductive signal has been measured as the voltage drop across a $50 \Omega$ load resistor.

observed for all materials at sufficiently high temperatures. In this case $[11,12]$ the characteristic field is given by $E_{c}=\left(3 m^{*} \hbar / \tau_{2}^{3} e^{2}\right)^{1 / 2}$ where $m^{*}$ is the effective mass of the carrier and $\tau_{2}=\hbar / 2 k_{B} T \pm \tau_{1}$ is the defect tunneling time. Here $\tau_{1}$ is of the order of the impurity vibration period, and the sign depends on the type of the impurity. The tunneling time $\tau_{2}$ as a function of $1 / T$ is shown in Fig. 2 for all materials.

In contrast to these observations which correspond to the quasistatic regime, the ionization gets frequency dependent and is drastically enhanced with rising frequency $\Omega$ when $\tau_{2}$ becomes comparable to $\Omega^{-1}$. This effect has been observed for various impurities in different semiconductors and is demonstrated for AlGaAs:Te in Fig. 1(b). The increase of $\tau_{2}$ is achieved by lowering the temperature (see Fig. 2).

The ionization probability still depends exponentially on the square of electric field strength, but with a substitution of the characteristic field $E_{c}$ by a field $E_{c}^{*}$, as $\exp \left[E^{2} /\left(E_{c}^{*}\right)^{2}\right]$ [see Fig. 1(b)]. The magnitude of $E_{c}^{*}$ now decreases strongly with increasing frequency in the range between 13 and $25 \mathrm{THz}$, whereas for $6.7 \mathrm{THz} E_{c}^{*}$ is the same as for $13 \mathrm{THz}$. For lower temperatures the ionization probability curves split at even lower frequencies. This is shown in Fig. 3 for Ge:Cu measured at $4.2 \mathrm{~K}$ where $\tau_{2}$ is largest for the present experimental conditions. This result for the frequency range between 6.8 and $3.4 \mathrm{THz}$ is obtained using the free electron laser.

In most cases deep impurities have one bound state which phenomenologically can be approximated by a

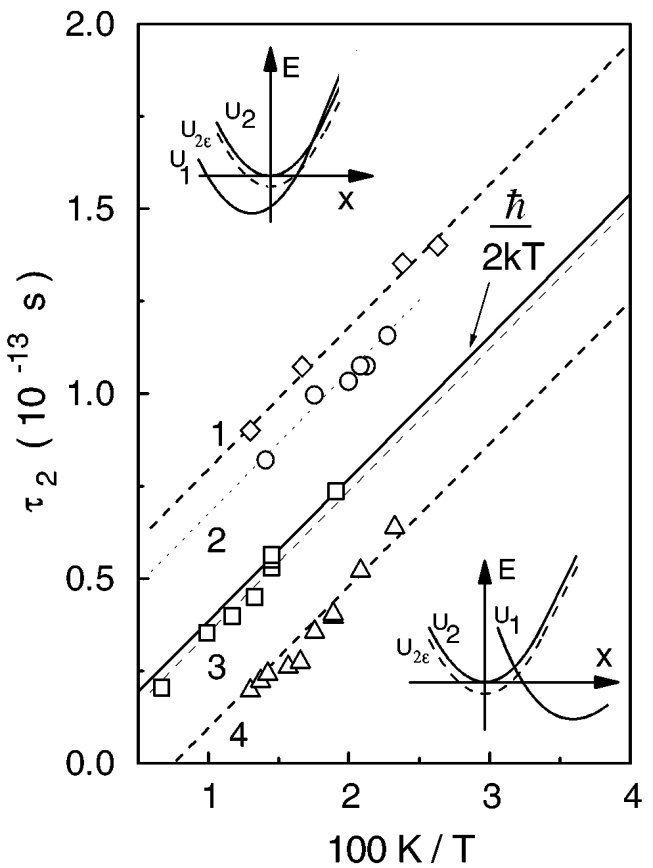

FIG. 2. Tunneling time $\tau_{2}$ determined from the experimental characteristic fields $E_{c}^{*}$ in the quasistatic regime as a function of inverse temperature. Frequency independence of $E_{c}^{*}$ can only be achieved at not too low temperatures limiting the temperature range where values of $\tau_{2}$ can be determined. $(\diamond) \mathrm{Ge}: \mathrm{Cu}$; (O) $\mathrm{Ge}: \mathrm{Hg}$; ( $\square$ ) $\mathrm{DX}^{-}$centers in $\mathrm{Al}_{0.35} \mathrm{Ga}_{0.65} \mathrm{As}$; $(\triangle) \mathrm{DX}^{-}$centers in $\mathrm{Al}_{0.5} \mathrm{Ga}_{0.5} \mathrm{Sb}$. Broken lines 1, 2, 3, and 4 show fits after $\tau_{2}=\hbar / 2 k T \pm \tau_{1}$ with $\tau_{1}=4.1 \times 10^{-14}, 2.9 \times 10^{-14}$, $0.3 \times 10^{-14}$, and $2.9 \times 10^{-14} \mathrm{~s}$, respectively. Insets show schematically adiabatic potential structures for substitutional impurities (top left) and for autolocalized impurities (bottom right).

potential well. The emission and capture of electrons or holes by deep impurities in semiconductors can be considered in the adiabatic approximation. Because of electron-phonon interaction the system consisting of local impurity vibrations and the electron is characterized by two adiabatic potentials $U_{1}(x)$ and $U_{2}(x)$ as a function of a configuration coordinate $x$. These adiabatic potentials correspond to the electron bound to the impurity and the electron detached from the impurity with zero kinetic energy, respectively (see insets of Fig. 2). The energy separation between the minima of $U_{1}$ and $U_{2}$ is the thermal ionization energy of the electron $\varepsilon_{T}$. Thermal emission of carriers from the bound state into the continuum is accomplished by thermal excitation of the system in the adiabatic bound state potential $U_{1}$ to an energy level $\mathcal{E}_{1}$ and tunneling of the impurity configuration from this state into the ionized configuration $U_{2}$ with energy $\mathcal{E}_{2} . \mathcal{E}_{1}$ and $\mathcal{E}_{2}$ are local vibrational energies of the impurity counted from the bottom of the corresponding potential energy parabola.

In the presence of an electric field the electron can be emitted at a negative kinetic energy $-\varepsilon$ due to tunneling through the triangular potential barrier formed from the electron potential well by the electric field. Hence 


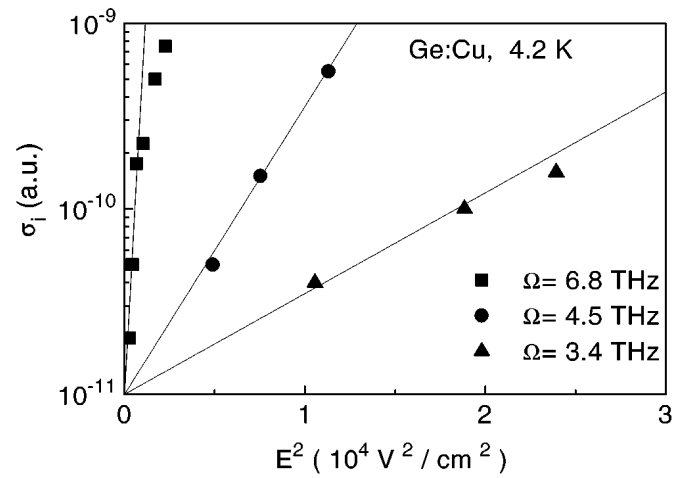

FIG. 3. Dependence of conductivity under irradiation $\sigma_{i}$ of $\mathrm{Ge}: \mathrm{Cu}$ at $4.2 \mathrm{~K}$ on the square of the amplitude of the electric field of the radiation. The photoconductive signal has been measured under constant voltage bias.

the adiabatic potential of the ionized configuration $U_{2}$ is shifted to lower energy $U_{2 \varepsilon} \equiv U_{2}-\varepsilon$. Thus electron emission in an electric field is achieved by two tunneling processes, electron tunneling with probability $P_{e}$ and tunneling of the defect between the configurations $U_{1}(x)$ and $U_{2 \varepsilon}$ with the probability $P_{d}$. Conservation of energy of the system during the process of defect tunneling gives $\mathcal{E}_{1}-\varepsilon_{T}=\mathcal{E}_{2}-\varepsilon$.

The ionization probability may be written as

$$
e(E)=\iint P_{e} P_{d} \exp \left(-\mathcal{E}_{1} / k_{B} T\right) d \varepsilon d \mathcal{E}_{1},
$$

where $T$ is the temperature and $k_{B}$ is the Boltzmann constant. The Boltzmann factor takes into account the thermal excitation of the system in the adiabatic potential $U_{1}$.

In the quasiclassical approximation, neglecting preexponential factors, the defect tunneling probability is given by $[13,14]$

$$
\begin{gathered}
P_{d} \propto \exp \left[-2\left(S_{2} \mp S_{1}\right)\right] \\
S_{i}\left(\mathcal{F}_{i}\right)=\frac{\sqrt{2 M}}{\hbar} \int_{a_{i}}^{x_{c}} d x \sqrt{U_{i}(x)-\mathcal{F}_{i}},
\end{gathered}
$$

where $i=1,2, M$ is a mass corresponding to the mode of impurity vibration, $a_{i}$ is the turning point in potential $U_{i}$ at the vibration energy $\mathcal{F}_{i}, x_{c}$ is the crossing point of potentials $U_{1}$ and $U_{2 \varepsilon}$, and the minus and plus sign is related to the particular impurity configuration shown in Fig. 2 top left and bottom right, respectively.

Electron tunneling in alternating electric fields has been theoretically treated by Keldysh [5] (see also [6]). In the limit $\varepsilon \gg \hbar \Omega$ the tunneling probability is given by

$$
P_{e} \propto \exp \left[-2 S_{e}(\varepsilon)\right]
$$

where

$$
S_{e}(\varepsilon)=-\frac{\varepsilon}{\hbar} \int_{0}^{\tau_{e}} \frac{1}{\gamma^{2}} \sinh ^{2}(\Omega \tau) d \tau+\frac{\varepsilon \tau_{e}}{\hbar},
$$

$$
\gamma \equiv \sqrt{2 m^{*} \varepsilon} \Omega / e E ; \quad \sinh \left(\Omega \tau_{e}\right)=\gamma .
$$

Here $m^{*}$ and $e$ are the electron effective mass and charge, correspondingly, and $\tau_{e}=\hbar \partial S_{e} / \partial \varepsilon$ has the meaning of an electron tunneling time.

The integral (1) has been calculated using the saddle point method. In the case of weak electric fields, i.e., as long as saddle point energy $\varepsilon \ll \varepsilon_{T}$, the exponent in the index can be developed into a power series of $\varepsilon$. Taken into account that $\mathcal{F}_{2}=\mathcal{F}_{1}-\left(\varepsilon_{T}-\varepsilon\right)$ we obtain

$$
\left.S_{2} \mp S_{1} \simeq\left(S_{2} \mp S_{1}\right)\right|_{\varepsilon=0}-\frac{\varepsilon \tau_{2}}{\hbar} ; \quad \tau_{2}=\left.\frac{\hbar \partial\left|S_{2}\right|}{\partial \mathcal{E}_{2}}\right|_{\varepsilon=0},
$$

then the saddle point condition gives

$$
\tau_{2}=\tau_{e} ; \quad \tau_{2}=\frac{\hbar}{2 k_{B} T} \pm \tau_{1},
$$

where $\tau_{1}=\hbar \partial\left|S_{1}\right| /\left.\partial \mathcal{E}_{1}\right|_{\varepsilon=0}$. The first equation in (7) states that the electron tunneling time is equal to the defect tunneling time for tunneling under the potential $U_{2}$.

Finally, we obtain for the ionization probability

$$
e(E) \propto \exp \left[\frac{E^{2}}{\left(E_{c}^{*}\right)^{2}}\right] \text { with }\left(E_{c}^{*}\right)^{2}=\frac{3 m^{*} \hbar}{e^{2}\left(\tau_{2}^{*}\right)^{3}}
$$

and

$$
\left(\tau_{2}^{*}\right)^{3}=\frac{3}{4 \Omega^{3}}\left[\sinh \left(2 \Omega \tau_{2}\right)-2 \Omega \tau_{2}\right] .
$$

This result shows that $\left(\tau_{2}^{*}\right)^{3}$ increases exponentially as a function of $\Omega \tau_{2}$. As shown in Eq. (8) the ionization probability itself depends exponentially on the third power of $\tau_{2}^{*}$, and tunneling ionization is drastically enhanced with rising $\Omega \tau_{2}$. This can be achieved not only by increasing the radiation frequency $\Omega$ but also by lowering the temperature [Eq. (7)]. This shows that in the limit $\Omega \tau_{2} \ll 1$ the time constant $\tau_{2}^{*}$ approaches the tunneling time $\tau_{2}$ giving the result of the quasistatic regime.

In Fig. 4 the ratio $\tau_{2}^{*} / \tau_{2}$ calculated after Eq. (9) is plotted as a function of $\Omega \tau_{2}$ and compared to experimental results obtained from measured values of $E_{c}^{*}$ for various frequencies, temperatures, and all the investigated materials. The tunneling times $\tau_{2}$ were determined from frequency independent values of $E_{c}^{*}$. Note that for the case of $\mathrm{Ge}: \mathrm{Cu}$ at $4.2 \mathrm{~K}$ independence of $E_{c}^{*}$ on frequency has not been observed up to the lowest frequency used, and therefore the value of $\tau_{2}$ has been calculated from its temperature dependence on the basis of the results given in Fig. 2 and Eq. (7). The experimental results shown in Fig. 4 are grouped after the materials. For each material the variation of the value of $\Omega \tau_{2}$ has been obtained by applying different radiation frequencies in the range from 6.7 to $54 \mathrm{THz}$ and different temperatures between 40 and $150 \mathrm{~K}$. For $\mathrm{Ge}: \mathrm{Cu}$ additional data are presented for $4.2 \mathrm{~K}$ and the frequency range from 3.4 to $6.8 \mathrm{THz}$. The plot 


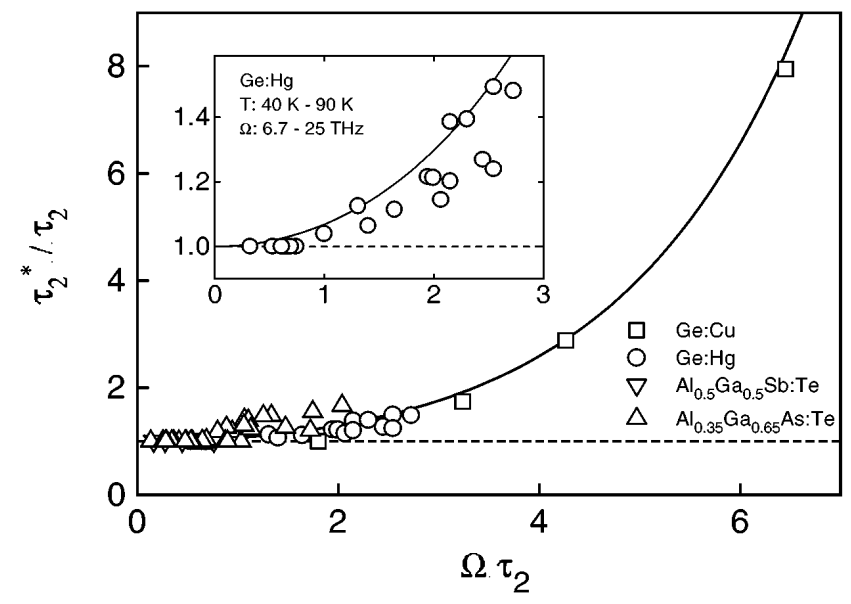

FIG. 4. The ratio $\tau_{2}^{*} / \tau_{2}$ as a function of $\Omega \tau_{2}$ calculated after Eq. (8) and obtained from measured values of $E_{c}^{*}$ for various frequencies, temperatures, and all investigated materials. The tunneling times $\tau_{2}$ are determined from frequency independent values of $E_{c}^{*}$. To show in detail the transition from the quasistatic regime $\Omega \tau_{2}<1$ to the high-frequency regime $\Omega \tau_{2}>1$ the data obtained for Ge:Hg are plotted in the inset for $\Omega \tau_{2}$ on an exaggerated scale. Note that even a ratio $\tau_{2}^{*} / \tau_{2}$ slightly larger than one causes a considerable enhancement of tunneling in an alternating field as compared to static fields, because the tunneling probability depends exponentially on the third power of tunneling time [Eq. (8)].

shows the theoretical result after Eq. (9) and experimental data which are in good agreement. It should be pointed out that the theory does not contain any fitting parameter. In particular, the strong enhancement of tunneling ionization and the quasistatic regime, where the ionization probability is independent of frequency, are clearly observed.

The tunneling process is controlled by $\Omega \tau_{2}$ where the tunneling time $\tau_{2}$ depends on temperature but also on the details of the adiabatic potential configuration. Basically the tunneling time is smaller for autolocalized centers with strong electron-phonon coupling (see Fig. 3) than for substitutional impurities with weak electron-phonon coupling. Thus higher frequencies and/or lower temperatures are needed to observe an enhancement of the tunneling emission for autolocalized centers. This is demonstrated with results on $\mathrm{AlGaSb}: \mathrm{Te}$ for which in the whole frequency and temperature range of the present investigation $\Omega \tau_{2}<1$ and the electron emission probability is independent of frequency (Fig. 4).

In summary, an enhancement of tunneling has been observed in alternating electric fields. In contrast to the quasistatic regime, where the electron tunnels at constant energy in a time much shorter than $\Omega^{-1}$, in the highfrequency regime the electron can absorb energy from the radiation field during tunneling leaving the barrier at higher energy. By this the effective width of the tunneling barrier is reduced and, thus, the tunneling probability enhanced. Typically the limit $\Omega \tau_{2}=1$ is in the range of terahertz frequencies where high electric fields can easily be applied in a contactless manner by powerful far-infrared lasers. Our measurements have been carried out with deep impurities in semiconductors; however, because tunneling is crucial in numerous processes in physics, chemistry, and biology, we expect that an enhancement of tunneling by contactless application of coherent radiation will have significant consequences.

Financial support by the DFG and a grant from the RFFI are gratefully acknowledged. S.G. thanks A. Belyaev for providing the AlGaAs samples. I.W. and E.H. thank J.N. Heyman and M.S. Sherwin for access to the FEL facility and active help.

*Present address: Institut für Angewandte Physik, Universität Hamburg, 20355 Hamburg, Germany.

[1] P.S. S. Guimaraes, B. J. Keay, J. P. Kaminski, S. J. Allen, Jr., P. F. Hopkins, A.C. Gossard, L. T. Florez, and J.P. Harbinson, Phys. Rev. Lett. 70, 3792 (1993).

[2] B. J. Keay, S. J. Allen, Jr., J. Gallan, J.P. Kaminski, K. L. Campman, A. C. Gossard, U. Bhattacharya, and J. W. Rodwell, Phys. Rev. Lett. 75, 4098 (1995).

[3] C. J. G. M. Langerak, B. N. Murdin, B. E. Cole, J. M. Chamberlain, M. Henini, M. Pate, and G. Hill, Appl. Phys. Lett. 67, 3453 (1995).

[4] S. D. Ganichev, W. Prettl, and P. G. Huggard, Phys. Rev. Lett. 71, 3882 (1993).

[5] L. V. Keldysh, Zh. Eksp. Teor. Fiz. 47, 1945 (1964) [Sov. Phys. JETP 20, 1307 (1965)].

[6] L.D. Landau and E.M. Livshitz, Quantum Mechanics (Pergamon, Oxford, 1977), p. 287.

[7] M. Büttiker and R. Landauer, Phys. Rev. Lett. 49, 1739 (1982).

[8] B. I. Ivlev and V. I. Mel'nikov, Phys. Rev. Lett. 55, 1614 (1985).

[9] M. V. Ammosov, N. B. Delone, and V.P. Krainov, Zh. Eksp. Teor. Fiz. 91, 2008 (1986) [Sov. Phys. JETP 64, 1191 (1986)].

[10] R. Landauer and Th. Martin, Rev. Mod. Phys. 66, 217 (1994).

[11] S. D. Ganichev, J. Diener, I. N. Yassievich, W. Prettl, B. K. Meyer, and K. W. Benz, Phys. Rev. Lett. 75, 1590 (1995).

[12] S. D. Ganichev, I. N. Yassievich, and W. Prettl, Semicond. Sci. Technol. 11, 679 (1996).

[13] T. Markvart, J. Phys. C 17, 6303 (1984).

[14] V.N. Abakumov, V.I. Perel, and I. N. Yassievich, in Nonradiative Recombination in Semiconductors, edited by V. M. Agranovich and A. A. Maradudin, Modern Problems in Condensed Matter Sciences Vol. 33 (North-Holland, Amsterdam, 1991). 